\title{
Performance Evaluation of Interacting Multiple Model Kalman Filter
}

\author{
VPS NAIDU AND J R RAOL \\ Multi Sensor Data Fusion Lab, Flight Mechanics and Control Division \\ National Aerospace Laboratories, Bangalore 560017 \\ email : vpsnaidu@gmail.com
}

\begin{abstract}
Conventional Kalman filter having constant velocity model (KFcv), conventional Kalman filter having constant acceleration model (KFca) and Interacting Multiple Model Kalman filter (IMMKF) having constant velocity \& constant acceleration models were implemented and their performance was studied. Overall performance of IMMKF was better than the other two. The performance of IMMKF with different process noise variance, measurement noise variance and model transition matrix was explored. It was concluded that Q1 and Q2 higher than their true would give better performance. The measurement noise variance $R$ lesser than the true value gives improved results. The diagonal elements of model transition matrix derived from sojourn time shows superior performance. Results showed that, IMMKF exhibits overall better performance and was more suitable for tracking maneuvering targets than conventional Kalman filters.
\end{abstract}

\section{INTRODUCTION}

$\mathbf{T}$ ARGET tracking problem is more difficult if the target is maneuvering. The Kalman filter [1-6] has some ability to adapt to maneuvers by tuning the Kalman filter to the most stressing maneuver expected. However, for targets that are not maneuvering or maneuvering at a level less than the most stressing maneuver, this approach results in less noise reduction than could be achieved with a Kalman filter tuned to a less stressing maneuver. In earlier multi target tracking systems, Kalman filter was used for a relatively benign maneuver to give adequate noise reduction when targets are not maneuvering, and the maneuver detector was used to adapt the filter to maneuvers and provide improved tracking performance through maneuver detection [712]. The problem encountered in practice with this approach is a time lag between the actual onset and the detection of the maneuver, and a time lag between the detection and the actual end of the maneuver. These lags typically last for several scans and large state errors can arise during these lags. In addition, random noise can trigger the maneuver detector and there is a time lag before switching back to the non-maneuver mode.

This relative insensitivity of the Kalman filter to target maneuvers has long been acknowledged and wide selections of methods have been developed to identify maneuvers and change the filter consequently. Of late, an innovative approach called the Interacting Multiple Model Kalman Filter (IMMKF) has been developed that seems to present superior performance to other approaches [13-17]. The IMMKF uses several target motion models (i.e. constant velocity, constant acceleration, co-ordinate turn model etc.) and has been successfully applied to track large maneuvering targets. IMMKF may use one model for straight and level flight

Paper No 107-B; Copyright (C) 2008 by the IETE. and different models for maneuvers or turns. The IMMKF always maintains all of the models and blends their outputs with weights that are computed probabilistically. In addition to the state estimates for each motion model, the IMMKF maintains an estimate of the probability that the target is moving in accordance with each model.

The tracking performance of conventional Kalman filter having constant velocity model (KFcv), conventional Kalman filter having constant acceleration model (KFca) and interacting multiple model Kalman filter (IMMKF) having both constant velocity and acceleration models are evaluated on the data of a simulated maneuvering target. Sensitivity of IMMKF to its design parameters is also investigated. The study made in MATLAB shows that IMMKF gives better overall performance than conventional $\mathrm{KFcv} \& \mathrm{KFca}$ and is, therefore, more suitable for tracking maneuvering targets. Next section provides a brief overview of target state estimation using IMMKF.

\section{IMMKF ALGORITHM}

The target tracking system involves the estimation of the state of a target. This could be done using the Kalman filter. Figure 1 shows the information flow diagram for one iteration of the Kalman filter. The estimation of the state of the target can be done in two steps [18].

Time prediction: forward prediction of the previous state $(\hat{X}(k \mid k))$ estimate to obtain the predicted estimate $(\tilde{X}(k+1 \mid k))$ for the current scan or frame

Measurement update: incorporating the current measurement $(z(k+1))$ into the predicted state estimate $(\tilde{X}(k+1 \mid k))$ to obtain an improved estimate 


\section{$(\hat{X}(k+1 \mid k+1))$}

The choice of the target motion model is one of the issues in the design of target tracking system. Kalman filter provides optimal estimation performance. However, this performance degrades rapidly when the target executes maneuvers. The more adaptable Interacting Multiple Model (IMM) has been proposed to deal with maneuvering targets. It is considered to be the most significant in target tracking since the Kalman filter.

IMMKF uses several possible models for the target's motion and a probabilistic switching between these models. The IMMKF is implemented with multiple parallel filters, where each of the filters corresponds to one of the assumed models. Due to the switching between models, there is exchange of information between filters. During each sampling period, all the filters of the IMMKF are in operation. The overall state estimate is a combination of the estimates from the individual filters. Let us consider $M_{1}, M_{2}, M_{3}, \ldots, M_{r}$ as the $r$ models of IMMKF and let $M_{j}(k)$ mean that the model $M_{j}$ is in effect during the sampling period ending at frame $k$. During the event $M_{j}(k+1)$ the state of the target evolves according to the following equation:

$$
X(k+1)=F_{j} X(k)+w_{j}(k)
$$

with the measurement given by:

$$
z(k+1)=H_{j} X(k+1)+v(k+1)
$$

where $F_{j}$ is the transition matrix and $H_{j}$ is the measurement matrix for $M_{j}(k+1)$. The process noise $w_{j}(k)$ and the measurement noise $v(k)$ are zero-mean, mutually independent, white, Gaussian processes with covariance matrices $Q_{j}(k)$ and $R(k)$ respectively. Figure 1 and Figure 2 show the information flow diagram for one iteration of the Kalman filter and IMMKF respectively. For simplicity, two model IMMKF is considered, i.e. $r=2$ and $j=1$ to $r$.

The IMMKF algorithm has following four major steps [13-17,19-21].

\subsection{Interaction/Mixing}

For the event $M_{j}(k+1)$, the mixed estimate $X_{0 j}(k \mid k)$ and the covariance $P_{0 j}(k \mid k)$ are computed as:

$$
\begin{array}{r}
\hat{X}_{0 j}(k \mid k)=\sum_{i=1}^{r} \mu_{i \mid j}(k \mid k) \hat{X}_{i}(k \mid k) \\
\hat{P}_{0 j}(k \mid k)=\sum_{i=1}^{r} \mu_{i \mid j}(k \mid k)\left\{\hat{P}_{i}(k \mid k)+\left[\hat{X}_{i}(k \mid k)-\hat{X}_{0 j}(k \mid k)\right]\right. \\
\left.\left[\hat{X}_{i}(k \mid k)-\hat{X}_{0 j}(k \mid k)\right]^{T}\right\}
\end{array}
$$

The mixing probabilities $\mu_{i \mid j}(k \mid k)$ are given by:

$$
\mu_{i \mid j}(k \mid k)=\frac{1}{\mu_{j}(k+1 \mid k)} p_{i j} \mu_{i}(k \mid k)
$$

where the predicted mode probability $\mu_{j}(k+1 \mid k)$ is computed by:

$$
\mu_{j}(k+1 \mid k)=\sum_{i=1}^{r} p_{i j} \mu_{i}(k \mid k)
$$

The mode switching process (usually Markov process) is specified by the following mode transition probabilities

$$
p_{i j}=\operatorname{Pr}\left\{M_{j}(k+1) \mid M_{i}(k)\right\}
$$

where $\operatorname{Pr}\{$.$\} denotes the probability of an event, which$ means $p_{i j}$ is the probability that $M_{i}$ model at $\mathrm{k}^{\text {th }}$ instant is switching over to $M_{j}$ model at $(k+1)^{\text {th }}$ instant. This is used to calculate the model probabilities for the final output.

\subsection{Kalman Filtering}

The usual Kalman filter equations are used with appropriate target motion models to update the mixed state estimates with current measurement as shown in Fig 2.

In each filter, the predictive part consists of the following equations [17,18].

Predicted state: $\tilde{X}_{j}(k+1 \mid k)=F_{j} \hat{X}_{j}(k \mid k)$

Predicted measurement:

$\tilde{z}_{j}(k+1 \mid k)=H_{j} \tilde{X}_{j}(k+1 \mid k)$

Predicted state covariance:

$\tilde{P}_{j}(k+1 \mid k)=F_{j} \hat{P}_{j}(k \mid k) F_{j}^{T}+G_{j} Q_{j} G_{j}^{T}$

Innovation covariance:

$S_{j}=H_{j} \widetilde{P}_{j}(k+1 \mid k) H_{j}^{T}+R$

The measurement update part consists of the following equations. 


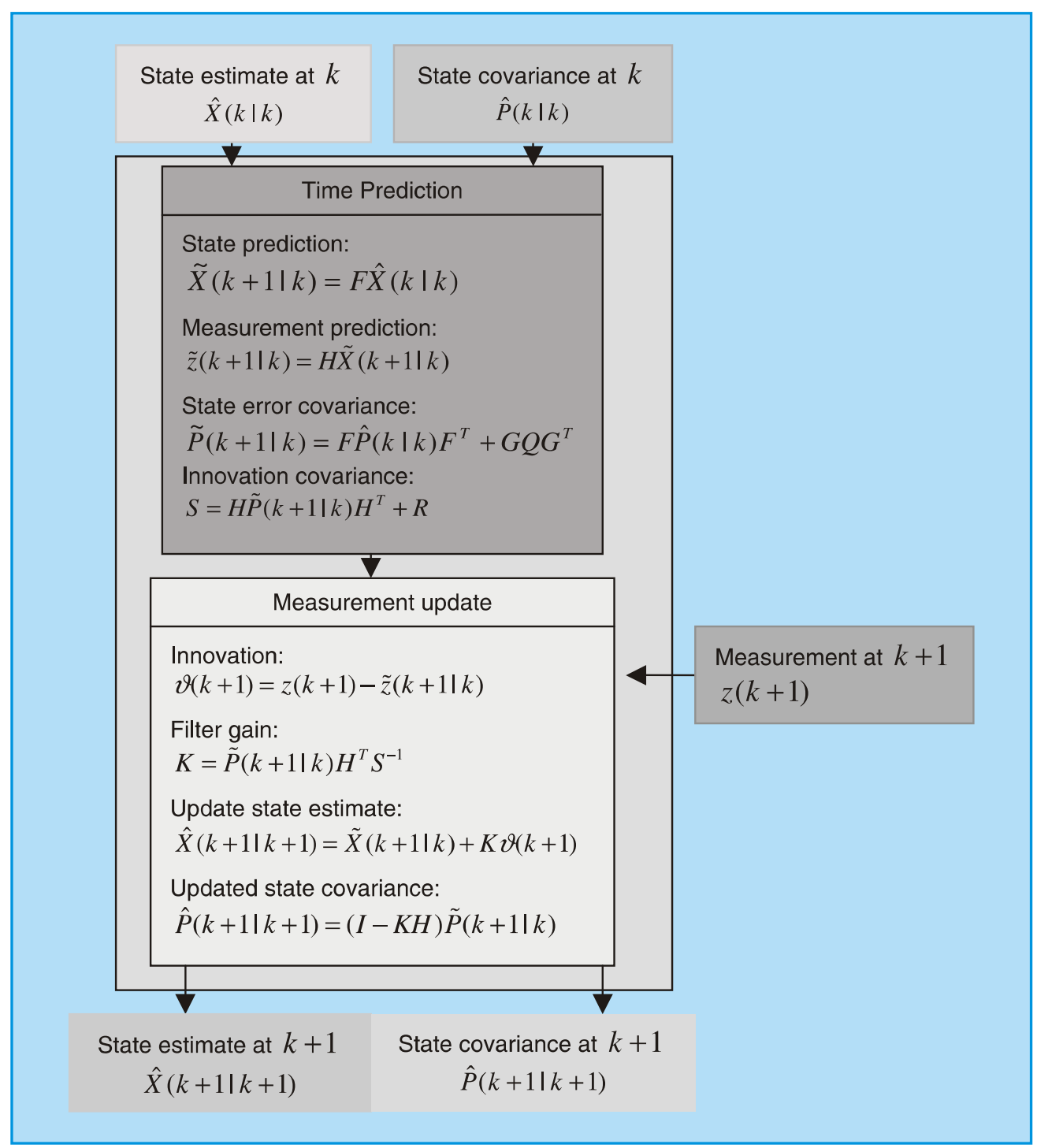

Figure 1 One iteration of the Kalman filter

Filter gain:

$$
K_{j}=\tilde{P}_{j}(k+1 \mid k) H_{j}^{T} S_{j}^{-1}
$$

Innovation:

$$
v_{j}=z(k+1)-\tilde{z}_{j}(k+1 \mid k)
$$

Updated state estimate:

$$
\hat{X}_{j}(k+1 \mid k+1)=\tilde{X}_{j}(k+1 \mid k)+K_{j} v_{j}
$$

Updated state covariance:

$$
\hat{P}_{j}(k+1 \mid k+1)=\left[I-K_{j} H_{j}\right] \tilde{P}_{j}(k+1 \mid k)
$$

Where $I$ is the unit matrix of size equal to state covariance matrix.

The likelihood function for matched filter $j$ is a Gaussian density function of innovation $v_{j}(k+1)$ with zero mean and innovation covariance $S_{j}$. It will be used to update the probabilities of the various models. It is computed as:

$$
\Lambda_{j}=\frac{1}{(2 \Pi)^{0.5 n} \sqrt{\left|S_{j}\right|}} \exp \left\{-0.5 v_{j}^{T} S_{j}^{-1} v_{j}\right\}
$$

where $n$ denotes the dimension of the innovation vector $u$.

\subsection{Mode Probability Update}

After each model has been updated with measurement $z(k+1)$, the mode probability $\mu_{j}(k+1 \mid k+1)$ is updated using mode likelihood's $\Lambda_{j}$ and the predicted mode probabilities $\mu_{j}(k+1 \mid k)$ for $M_{j}(k+1)$. 


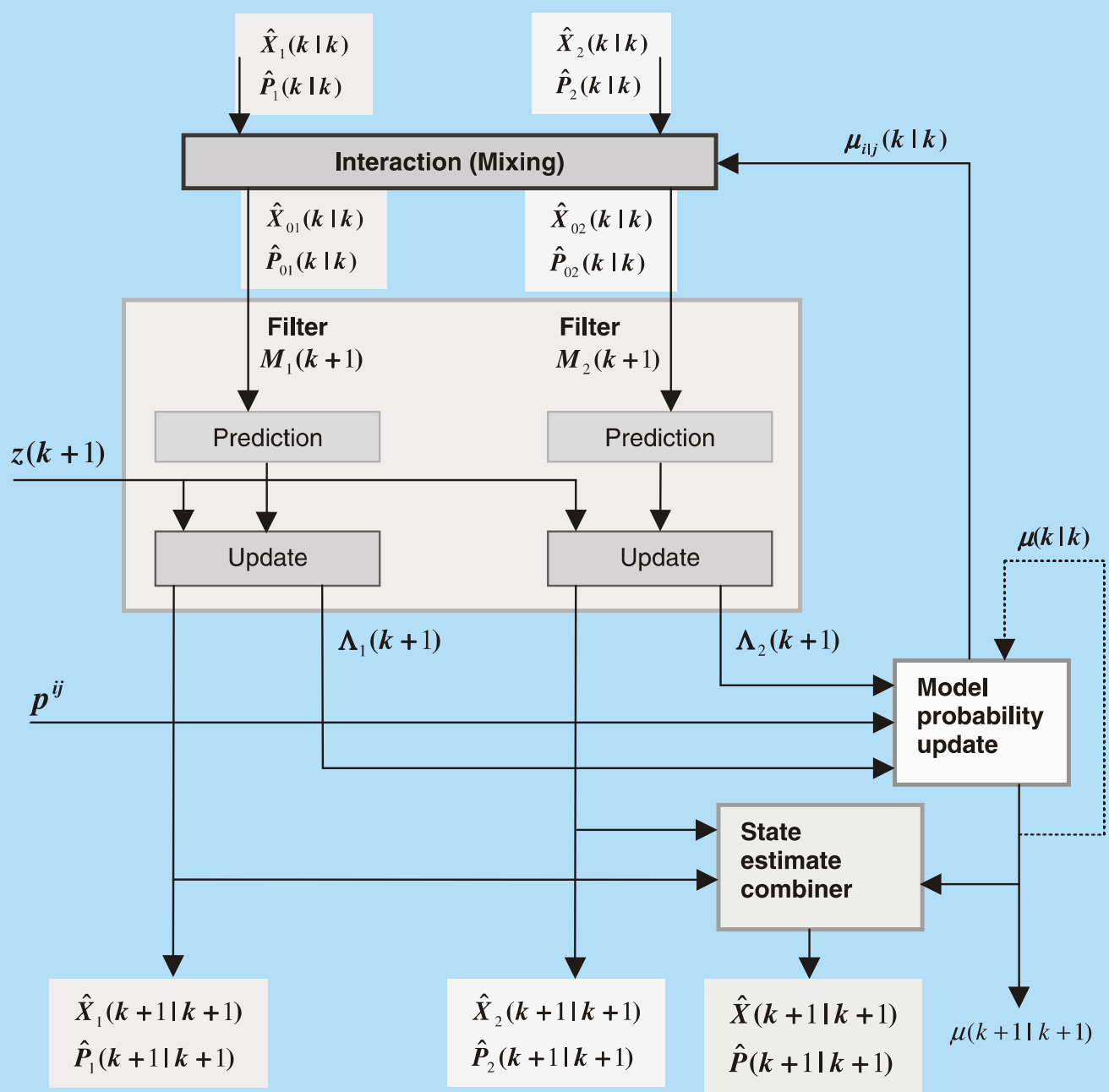

Figure 2 One iteration of the IMMKF

$$
\mu_{j}(k+1 \mid k+1)=\frac{1}{c} \mu_{j}(k+1 \mid k) \Lambda_{j}
$$

where the normalization factor

$$
c=\sum_{i=1}^{r} \mu_{i}(k+1 \mid k) \Lambda_{i}
$$

\subsection{State Estimate and Covariance Combiner}

Finally the estimated states $\hat{X}_{j}(k+1 \mid k+1)$ and covariance $\hat{P}_{j}(k+1 \mid k+1)$ from each filter are combined using the updated mode probability $\mu_{j}(k+1 \mid k+1)$ to produce overall state estimate $\hat{X}(k+1 \mid k+1)$ and the associated covariance $\hat{P}(k+1 \mid k+1)$ as given below:

$$
\hat{X}(k+1 \mid k+1)=\sum_{j=1}^{r} \mu_{j}(k+1 \mid k+1) \hat{X}_{j}(k+1 \mid k+1)
$$$$
\hat{P}(k+1 \mid k+1)=
$$

$$
\begin{aligned}
& \sum_{j=1}^{r} \mu_{j}(k+1 \mid k+1)\left\{\begin{array}{l}
\hat{P}_{j}(k+1 \mid k+1)+ \\
{\left[\hat{X}_{j}(k+1 \mid k+1)-\hat{X}(k+1 \mid k+1)\right]}
\end{array}\right. \\
& \left.\left[\hat{X}_{j}(k+1 \mid k+1)-\hat{X}(k+1 \mid k+1)\right]^{T}\right\}
\end{aligned}
$$

\section{TARGET MOTION MODELS}

The most common forms of target motion models are:

1. $2^{\text {nd }}$ order kinematic model (constant velocity model) 
2. $3^{\text {rd }}$ order kinematic model (constant acceleration model)

\subsection{Constant Velocity Model}

The $2^{\text {nd }}$ order kinematic model, with position and velocity components in each of the three Cartesian coordinates $x, y$ and $z$, has the following transition and process noise gain matrices:

$$
\begin{aligned}
F_{C V} & =\left[\begin{array}{ccc}
\Phi_{C V} & 0 & 0 \\
0 & \Phi_{C V} & 0 \\
0 & 0 & \Phi_{C V}
\end{array}\right] \\
G_{C V} & =\left[\begin{array}{ccc}
\varsigma_{C V} & 0 & 0 \\
0 & \varsigma_{C V} & 0 \\
0 & 0 & \varsigma_{C V}
\end{array}\right]
\end{aligned}
$$

where

$$
\Phi_{C V}=\left[\begin{array}{lll}
1 & T & 0 \\
0 & 1 & 0 \\
0 & 0 & 0
\end{array}\right] \quad \varsigma_{C V}=\left[\begin{array}{c}
T^{2} / 2 \\
T \\
0
\end{array}\right]
$$

In eqn (20), the variations in velocity are modeled as zero-mean white noise accelerations. Low noise variance $Q_{c v}$ is used with the model to represent the constant course and speed of the target in a nonmaneuvering mode. The process noise intensity in each coordinate is generally assumed to be small and equal $\left(\sigma_{x}^{2}=\sigma_{y}^{2}=\sigma_{z}^{2}\right.$ ), which accounts for air turbulence, slow turns, and small linear acceleration.

Although the $2^{\text {nd }}$ order model is primarily used to track the non-maneuvering mode of a target, use of higher level of process noise variance will allow the model to track maneuvering targets as well, albeit to a limited extent.

\subsection{Constant Acceleration Model}

The $3^{\text {rd }}$ order model, with position, velocity and acceleration components in each of the three Cartesian coordinates $\mathrm{x}, \mathrm{y}$ and $\mathrm{z}$ has the following transition and process noise gain matrices:

$$
\begin{aligned}
F_{C A} & =\left[\begin{array}{ccc}
\Phi_{C A} & 0 & 0 \\
0 & \Phi_{C A} & 0 \\
0 & 0 & \Phi_{C A}
\end{array}\right] \\
G_{C A} & =\left[\begin{array}{ccc}
\varsigma_{C A} & 0 & 0 \\
0 & \varsigma_{C A} & 0 \\
0 & 0 & \varsigma_{C A}
\end{array}\right]
\end{aligned}
$$

where

$$
\Phi_{C A}=\left[\begin{array}{ccc}
1 & T & T^{2} / 2 \\
0 & 1 & T \\
0 & 0 & 1
\end{array}\right] \quad \varsigma_{C A}=\left[\begin{array}{c}
T^{3} / 6 \\
T^{2} / 2 \\
T
\end{array}\right]
$$

The acceleration increments over a sampling period are a discrete time zero-mean white noise. A low value of process noise variance $Q_{2}$ (but relatively higher than $Q_{1}$ ) will yield nearly a constant acceleration motion. The noise variances in each coordinate are assumed to be equal $\left(\sigma_{x}^{2}=\sigma_{y}^{2}=\sigma_{z}^{2}\right)$.

Studies have shown that use of higher process noise levels combined with $3^{\text {rd }}$ order kinematic model can help track the onset and termination of a maneuver to a certain extent.

\subsection{IMMKF Algorithm}

The algorithm that is being developed in MATLAB consists of both IMMKF and conventional KF. Two model IMMKF (non-maneuver mode and maneuver mode i.e. $r=2$ ) is considered to describe the principles and steps of IMMKF algorithm.

The observation matrix

$$
H=\left[\begin{array}{lllllllll}
1 & 0 & 0 & 0 & 0 & 0 & 0 & 0 & 0 \\
0 & 0 & 0 & 1 & 0 & 0 & 0 & 0 & 0 \\
0 & 0 & 0 & 0 & 0 & 0 & 1 & 0 & 0
\end{array}\right]
$$

The initial mode probabilities $\mu=\left[\begin{array}{ll}\mu_{1} & \mu_{2}\end{array}\right]^{T}$ corresponding to non-maneuver and maneuver mode can be taken as 0.9 and 0.1 respectively.

$p_{i j}$ is the Markov transition matrix, which takes care of the switching from mode $i$ to mode $j$. This is a design parameter and is chosen by the user [19-22]. The switching probabilities are generally known to depend upon sojourn time. For example, consider the following Markov chain transition matrix between the two modes of the IMMKF

$$
p_{i j}=\left[\begin{array}{cc}
0.9 & 0.1 \\
0.33 & 0.67
\end{array}\right]
$$

The basis for selecting $p_{12}=0.1$ is that, in the initial stage, the target is likely to be in non-maneuvering mode and the probability to switch over to maneuvering mode will be relatively low. On the other hand, $p_{22}$ is selected based on the number of sampling periods for 
which the target is expected to maneuver (sojourn time). If the target maneuver lasts for 3 sample periods ( $\tau=3$ ), the probability $p_{22}$ is given by:

$$
p_{22}=1-\frac{1}{\tau}=0.67
$$

The performance of the IMMKF with respect to conventional KFcv and KFca is checked by computing and plotting the following $[16,23]$ :

\section{Measured and estimated $x, y \& z$ positions}

2. Mean in $x, y \& z$ position error:

Mean in $x$ position error $=\frac{1}{N} \sum_{i=1}^{N}\left(x_{i}-\hat{x}_{i}\right)$

Similarly for $y$ and $z$ positions Where $N$ is the number of samples, $x$ is the measured position and is the estimated position.

3. The percentage fit error (PFE) in $x, y \& z$ positions:

PFE $x=100 * \frac{\operatorname{norm}(x-\hat{x})}{\operatorname{norm}(x)}$

Similar expressions for $y$ (PFEy) and $z$ positions (PFEz)

4. Root mean square position error:

RMSPE $=\sqrt{\frac{1}{N} \sum_{i=1}^{N} \frac{\left(x_{i}-\hat{x}_{i}\right)^{2}+\left(y_{i}-\hat{y}_{i}\right)^{2}+\left(z_{i}-\hat{z}_{i}\right)^{2}}{3}}$

Similar expressions for velocity (RMSVE) and accelerations (RMSAE)

5. Root sum square position error:

$$
\operatorname{RSSPE}=\sqrt{(x-\hat{x})^{2}+(y-\hat{y})^{2}+(z-\hat{z})^{2}}
$$

Similar expressions for velocity (RSSVE) and accelerations (RSSAE)

6. The root sum variance:

$\mathrm{RSvarP}=\sqrt{P_{x}+P_{y}+P_{z}}$

where $P_{x}, P_{y} \& P_{z}$ are the diagonal elements in $P$ corresponding to positions $x, y \& z$

\subsection{Validation with Simulated Data}

The data for maneuvering target is simulated using the $2^{\text {nd }}$ and $3^{\text {rd }}$ order models with sampling interval of 1 second and a total of 500 seconds. The simulation proceeds with the following parameters:

$>\quad$ Initial state $(x, \dot{x}, \ddot{x}, y, \dot{y}, \ddot{y}, z, \dot{z}, \ddot{z})$ of the target is (11097.6, -6.2, 0, 3425, -299.9, 0, 40, 0,0)

$>$ A low-level process noise variance of Q1 $=0.09$ is considered for model-1

$>$ A higher-level process noise variance of Q2=36 is considered for model-2

$>$ Noise variances for both models in each coordinate are assumed to be equal i.e.

$Q_{x x}=Q_{y y}=Q_{z z}=Q$

$>$ Measurement noise variance of $\mathrm{R}=100$ is considered, it is also assumed to be equal

$R_{x x}=R_{y y}=R_{z z}=R$

At scan 100 , additional acceleration $\ddot{x}=27.4 \mathrm{~m} / \mathrm{s}^{2}$ is given in $x$ directions to simulate first maneuver and at scan $350, \ddot{y}=-99.4 \mathrm{~m} / \mathrm{s}^{2}$ is given $y$ direction to simulate the second maneuver. The acceleration profiles in $x$ - and $y$-coordinate are shown in Fig 3. The target spends most of the time in non-maneuvering sate. The
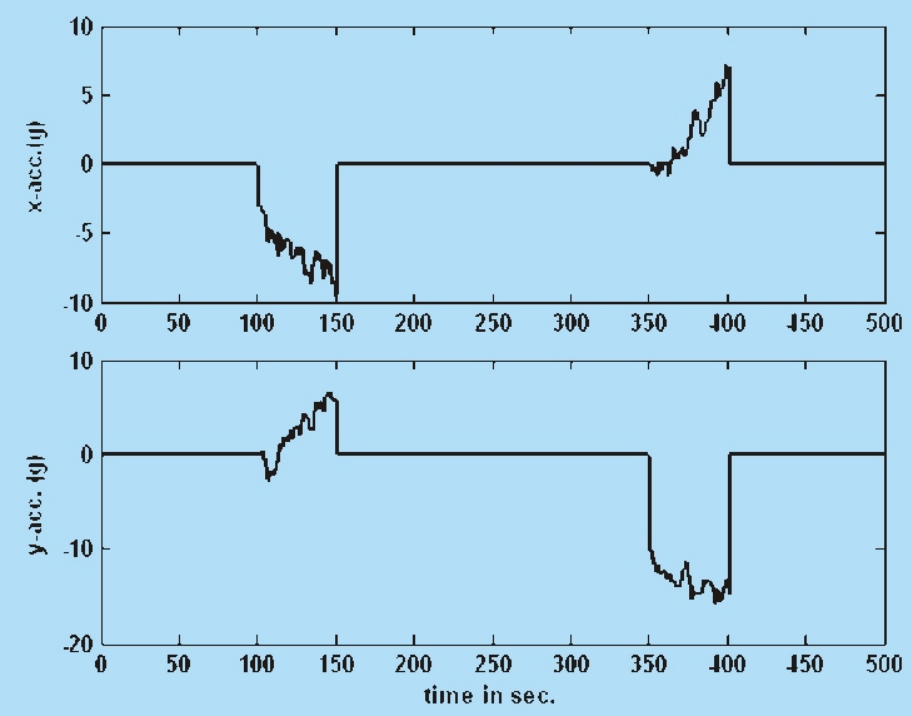

Figure 3 Acceleration profiles in $\mathrm{x}$ - and $\mathrm{y}$-axis 
first maneuver starts at scan 100 and ends at scan 150 and the second maneuver starts at scan 350 and ends at scan 400 .

KFcv uses a single constant velocity model with $\mathrm{Q} 1=0.09$ and $\mathrm{R}=100, \mathrm{KFca}$ uses a single constant acceleration model with $\mathrm{Q} 2=36$ and $\mathrm{R}=100$, and IMMKF with two mode (constant velocity model with Q1=0.09 $\& \mathrm{R}=100$ for non-maneuvering mode and constant acceleration model with $\mathrm{Q} 2=36$ \& $\mathrm{R}=100$ for maneuvering mode) filters to track the target through both the modes. The Markov chain transition matrix is,

$$
p_{i j}=\left[\begin{array}{cc}
0.9 & 0.1 \\
0.05 & 0.95
\end{array}\right]
$$

The above choice is made keeping in view the sojourn time $(\tau=50)$ of the target. Equation (22) is used to arrive at the $p_{22}$ value in the above transition matrices.

The true and estimated trajectories using KFcv, KFca and IMMKF are shown in Fig $4 a$. The enlarged view of some portion of Fig $4 a$ is shown in Fig $4 b$. It is observed that the track tracked by $\mathrm{KFcv}$ is away from the true track during the maneuver. The tracks tracked by both KFca and IMMKF are very close to the true track.

The mode probabilities are shown in Fig 5. It is clearly shown that there are two maneuvers in the trajectory with start and end time of the maneuvers. This indicates which model or the weighted combination of models is active at a given time.

The RSVar from $\mathrm{KFcv}, \mathrm{KFca}$ and IMMKF are shown in Fig 6. The RSvar in KFcv and KFca are

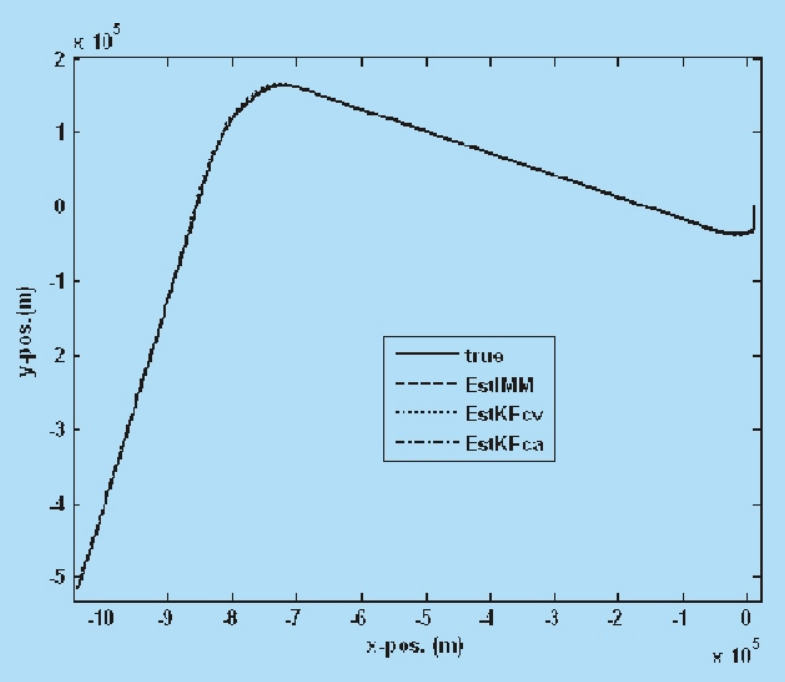

Figure 4a True and estimated trajectories

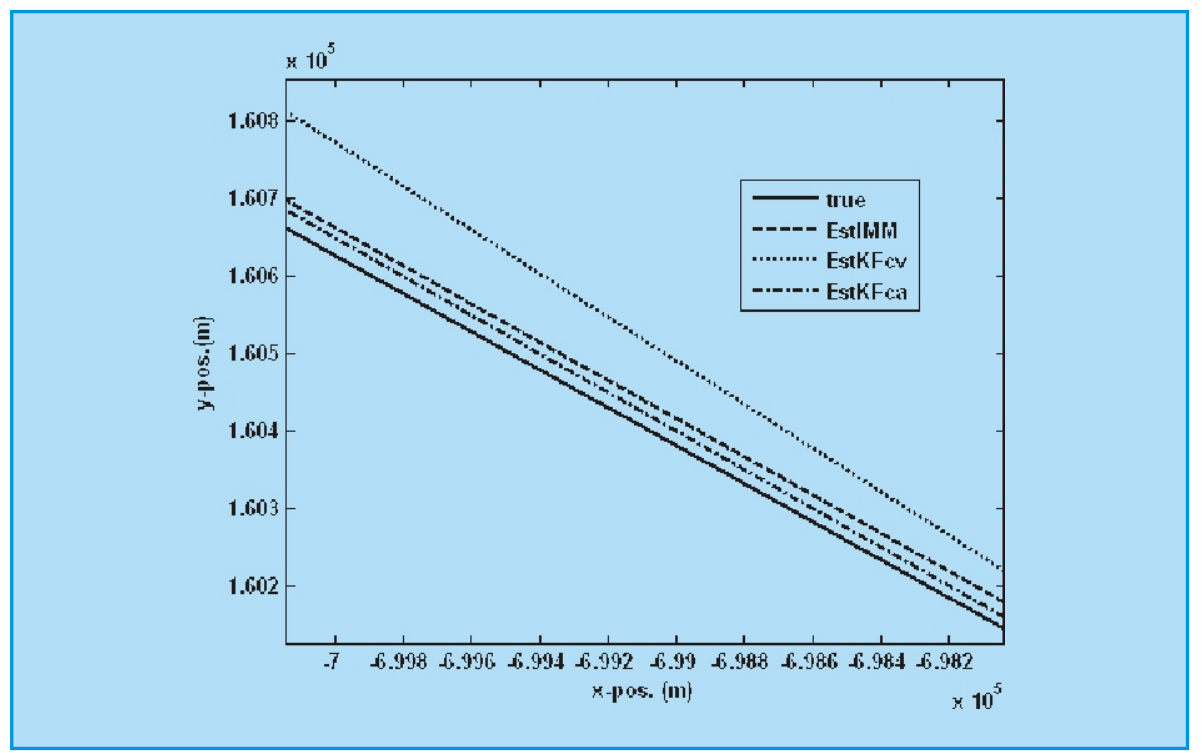

Figure 4b Enlarged view of some portion of Fig-4a 


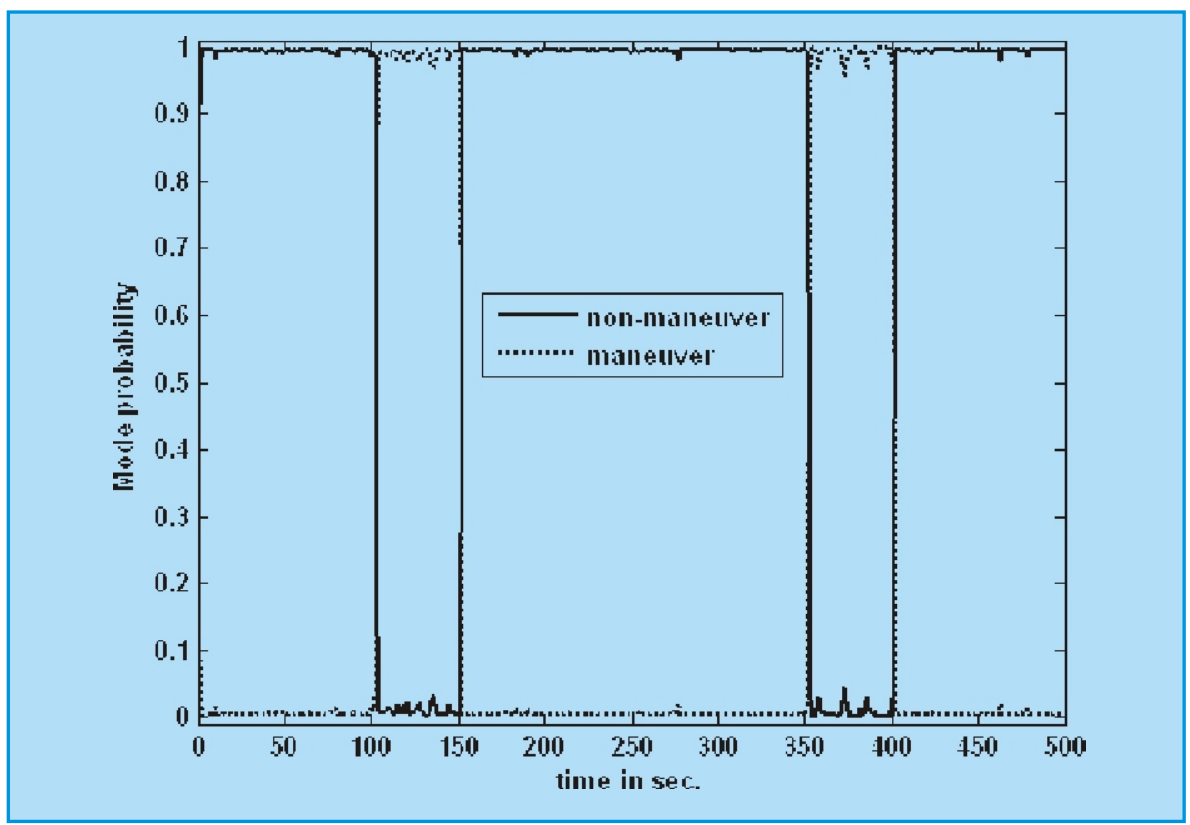

Figure 5 Mode probabilities

constant throughout the trajectory. Due to high process noise covariance the RSVar is high in KFca than KFcv. But it is high during maneuver and low during nonmaneuver in case of IMMKF. This information could be useful in track fusion and it is one of the features of IMMKF. This shows that maneuvers can detect using the IMMKF very well.

The RSSPE from KFcv, KFca and IMMKF are shown in Fig $7 a$. It is very high during the maneuver in case of KFcv. The enlarged view of Fig $7 a$ is shown in Fig $7 b$. It is low throughout the trajectory in case of KFca. The mean in $x^{-}, y-$ and $z$-position errors, PFE in $\mathrm{x}-, \mathrm{y}-$ and $\mathrm{z}$-positions are shown in Table 1. These results are obtained from 50 Monte Carlo simulations. From the Table and Figs, it is observed that the tracking performance is worst in case of $\mathrm{KFcv}$ during maneuver. The tracking performance of both KFca and IMMKF are almost similar during maneuver. In overall, the tracking performance of IMMKF is better.

Table 1 : Comparison of KFcv, KFca and IMMKF

\begin{tabular}{|l|l|l|l|l|l|l|}
\hline Filter & $P F E x$ & $P F E y$ & $P F E z$ & RMSPE & RMSVE & RMSAE \\
\hline KFcV & 0.11 & 0.84 & 0.11 & 824.4 & 220.78 & 29.49 \\
\hline KFca & 0 & 0 & 0 & 2.75 & 9.15 & 7.72 \\
\hline IMMKF & 0 & 0 & 0 & 2.78 & 6.88 & 5.83 \\
\hline
\end{tabular}

\subsection{IMMKF Performance}

The performance of IMMKF against the tuning parameters viz., process noise variances Q1, Q2, measurement noise variance $\mathrm{R}$ and model transition matrix $p_{i j}$ are discussed in the following section. All results are obtained from fifty Monte Carlo simulations.

\subsubsection{Effect of $Q 1$ on IMMKF}

The true $\mathrm{Q} 1$ is 0.09 . In this study, $\mathrm{Q} 1<$ true as 0.01 and $\mathrm{Q} 1>$ true as 0.19 are taken to evaluate its effect on the filter performance. RSVar is high as shown in Fig 8 for Q1>true and less for Q1<true during nonmaneuver phase and there is no change during maneuver phase. RMSPE, RMSVE and RMSAE are shown in Table 2. It is observed that $\mathrm{Q} 1>$ true gives marginally better results in terms of RMSPE and at the same time the RSvar is high that shows the increased uncertainty in state estimation during non-maneuver period.

Table 2 : Effect of $Q 1$ on IMMKF performance

\begin{tabular}{|l|l|l|l|}
\hline Parameter & Q1<true & Q1=true & Q1>true \\
\hline RMSPE & 2.21 & 2.19 & 2.12 \\
\hline RMSVE & 6.77 & 6.76 & 6.75 \\
\hline RMSAE & 5.80 & 5.80 & 5.80 \\
\hline
\end{tabular}

\subsubsection{Effect of $Q 2$ on $I M M K F$}

The true Q2 is 36. In this study, Q2<true as 18 and Q2>true as 54 are taken to evaluate its effect on the filter performance. RSVar is high for Q2>true and less for Q2<true during maneuver phase as shown in Fig 9. RMSPE, RMSVE and RMSAE are shown in Table 3. It is observed that $\mathrm{Q} 2>$ true provides improved results in terms of RMSPE and at the same time the uncertainty in the state estimation is high during maneuver period. It is because; high Q2 is specified in acceleration model that goes directly into the covariance computation (see (eq. 9)). 


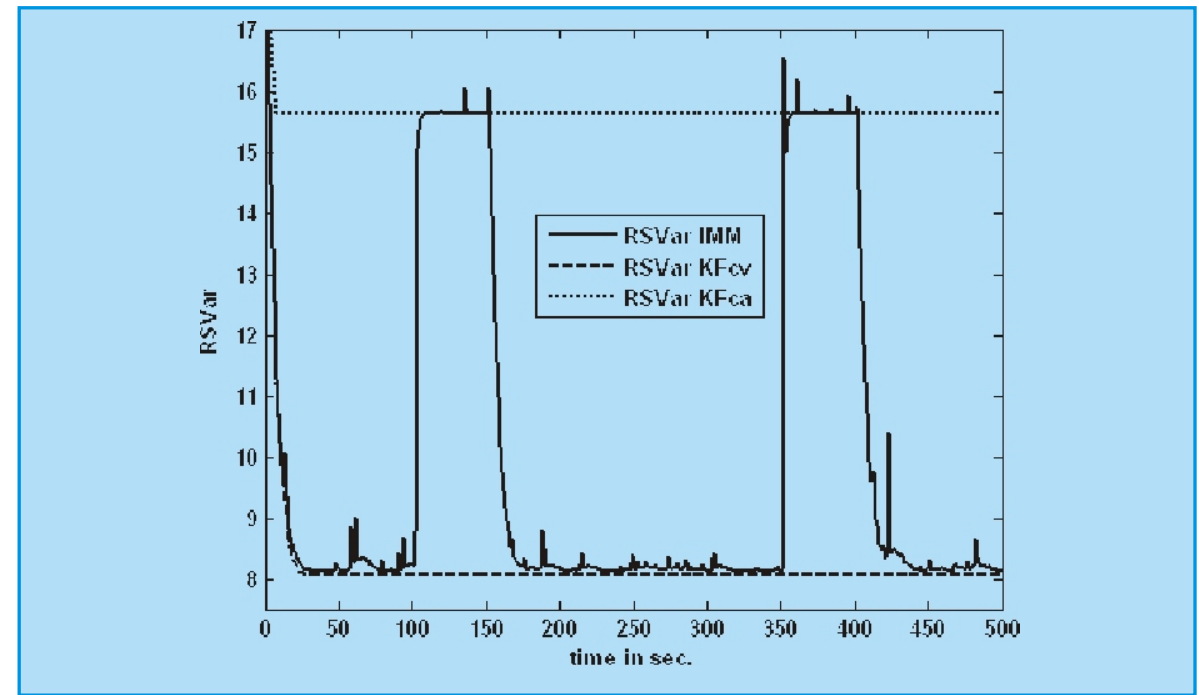

Figure 6 RSVar from $\mathrm{KFcv}$, KFca and IMM

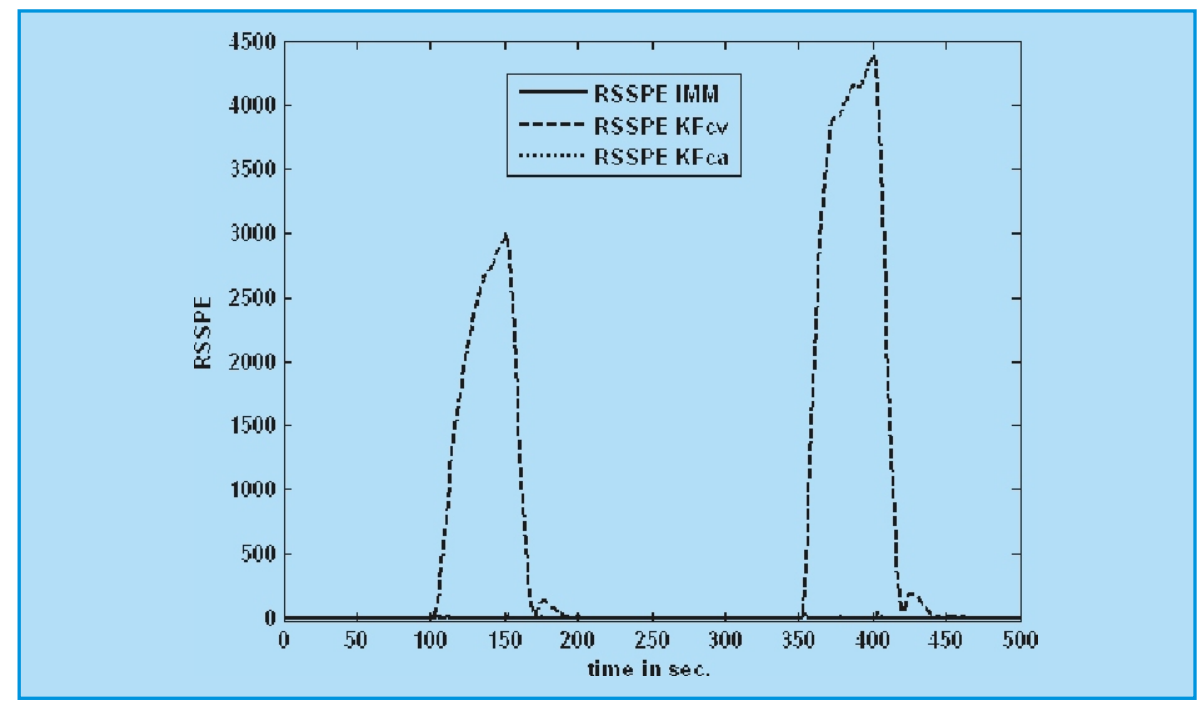

Figure 7a RSSPE from $\mathrm{KFcv}$, KFca and IMM

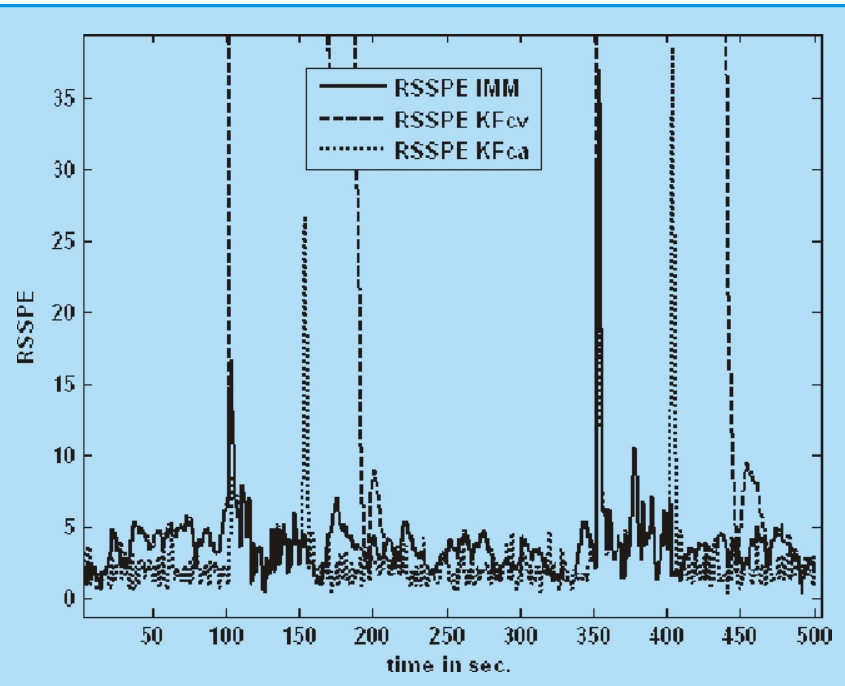

Figure 7b Enlarged view of some portion of Fig-7a 


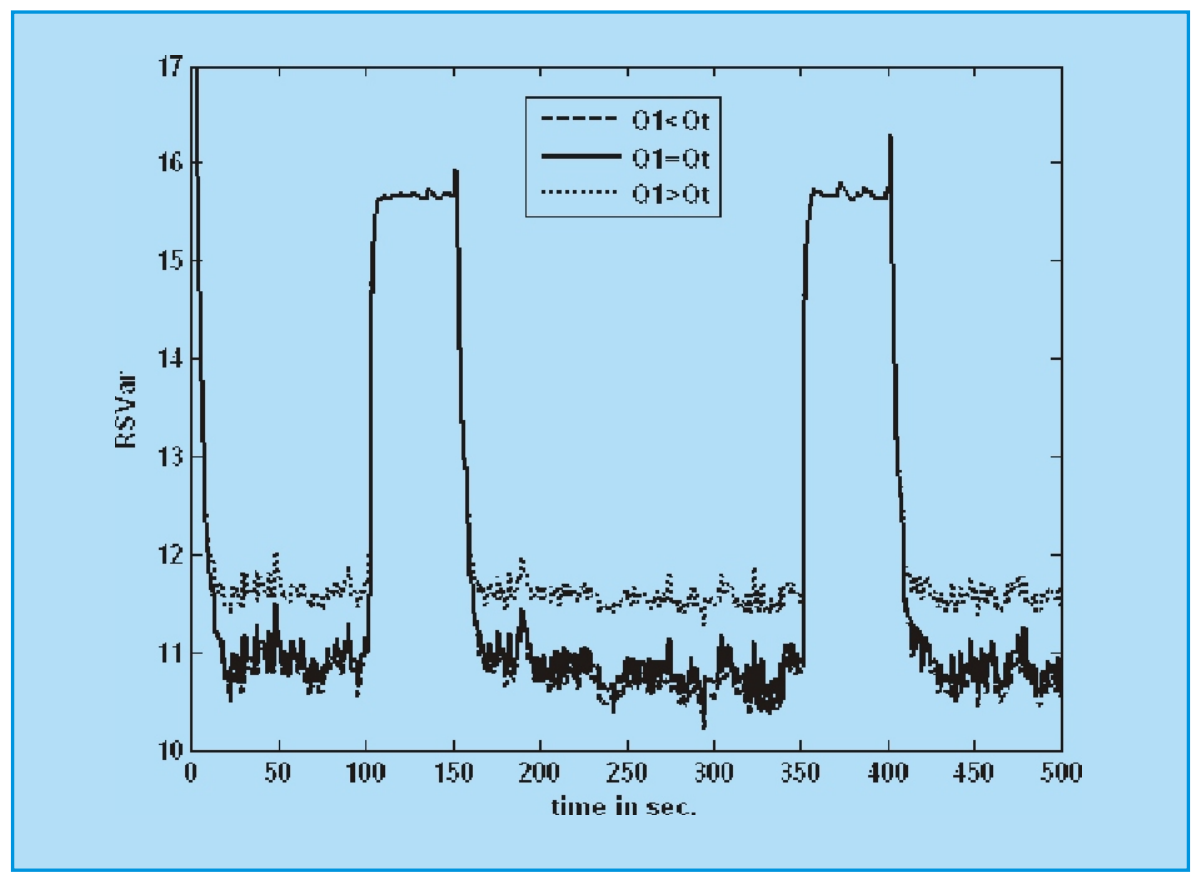

Figure 8 RSVar - different Q1

Table 3 : Effect of $Q 2$ on IMMKF performance

\begin{tabular}{|l|l|l|l|}
\hline Parameter & Q2<true & Q2=true & Q2>true \\
\hline RMSPE & 3.01 & 2.19 & 1.92 \\
\hline RMSVE & 7.71 & 6.76 & 6.40 \\
\hline RMSAE & 6.10 & 5.80 & 5.66 \\
\hline
\end{tabular}

\subsubsection{Effect of $R$ on IMMKF Performance}

The true $\mathrm{R}$ is 100 . In this study, $\mathrm{R}<$ true as 50 and Q2>true as 150 are taken to evaluate its effect on the tracker performance. Mode probabilities are changed with respect to change in $\mathrm{R}$ as shown in Fig 10. The RSVar is high for $\mathrm{R}>$ true throughout the trajectory as shown in Fig 11. RMSPE, RMSVE and RMSAE are shown in Table 4. It is observed that $\mathrm{R}<$ true provides better results and the uncertainty in the target state estimation is also improved. It is because; small $\mathrm{R}$ increases the Kaman gain $\mathrm{K}$ that reduces the covariance (see (eqn 10), (eqn 11) \& (eqn 14)).

Table 4 : Effect of $R$ on IMMKF performance

\begin{tabular}{|l|lll|}
\hline Parameter & $\mathrm{R}<$ true & $\mathrm{R}=$ true & $\mathrm{R}>$ true \\
\hline RMSPE & 1.63 & 2.19 & 2.74 \\
\hline RMSVE & 5.94 & 6.76 & 7.46 \\
\hline RMSAE & 5.12 & 5.80 & 6.29 \\
\hline
\end{tabular}

\subsubsection{Effect of model transition matrix $\left(p_{i i}\right)$ on IMMKF}

The true diagonal elements of $p_{i i}$ are 0.98 . In this study, $p_{i i}<$ true as 0.7 and Q2>true as 0.999 are taken to evaluate its effect on the performance. There are changes in mode probabilities with respect to changes in $p_{i i}$ as shown in Fig 12. RSVar is low for $p_{i i}>$ true and high for $p_{i i}<$ true during non-maneuver phase as shown in Fig 13 and it is little bit high for $p_{i i}<$ true during maneuver phase. RMSPE, RMSVE and RMSAE are shown in Table 5. It is observed that $p_{i i}$ computation based on sojourn time provides better results. The uncertainty in target state estimation could be improved by increasing the diagonal elements of $p_{i i}$.

Table 5 : Effect of $p_{i i}$ on IMMKF performance

\begin{tabular}{|l|l|c|c|}
\hline Parameter & $\mathrm{p}<$ true & $\mathrm{p}=$ true & $\mathrm{p}>$ true \\
\hline RMSPE & 2.18 & 2.19 & 2.46 \\
\hline RMSVE & 6.88 & 6.76 & 7.13 \\
\hline RMSAE & 6.10 & 5.80 & 6.39 \\
\hline
\end{tabular}

RMSPE, RMSVE and RMSAE for different parameters are (shown in Table 1 to 5) presented in bar graph as shown in Fig 14. It is observed that Q1 and Q2 higher than their true will give better performance. The measurement noise variance $\mathrm{R}$ lesser than the true value and the diagonal elements of model transition matrix derived from sojourn time give better performance. 


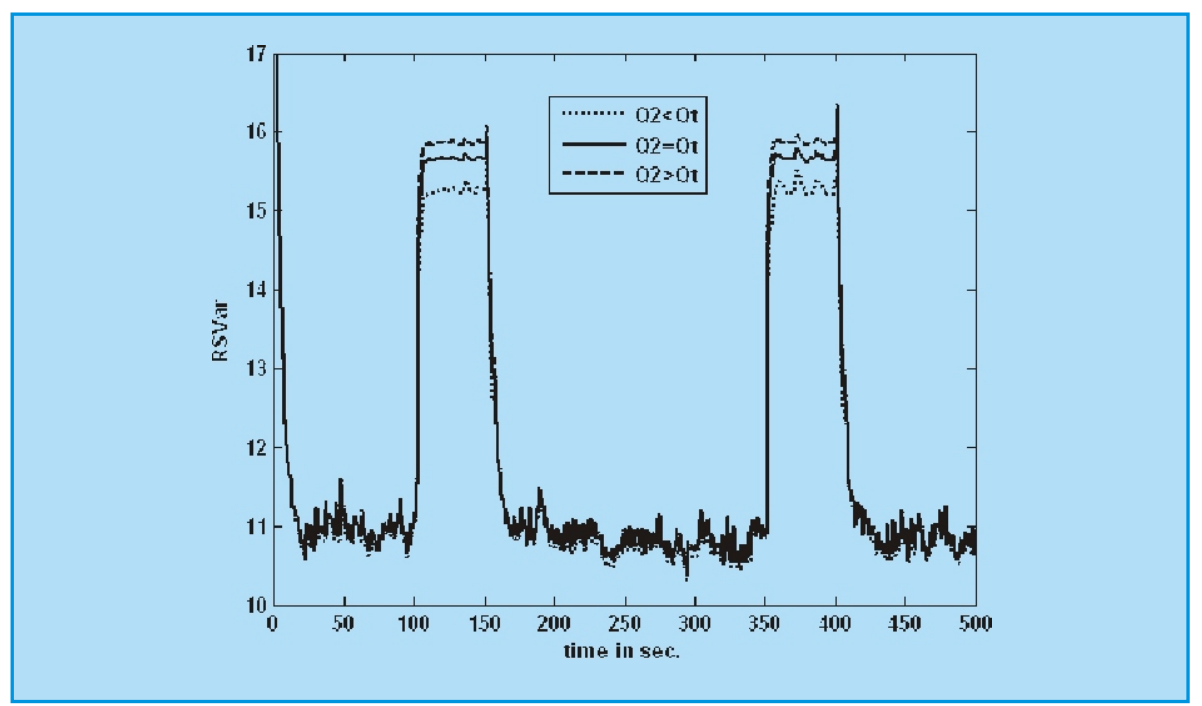

Figure 9 RSVar - different Q2

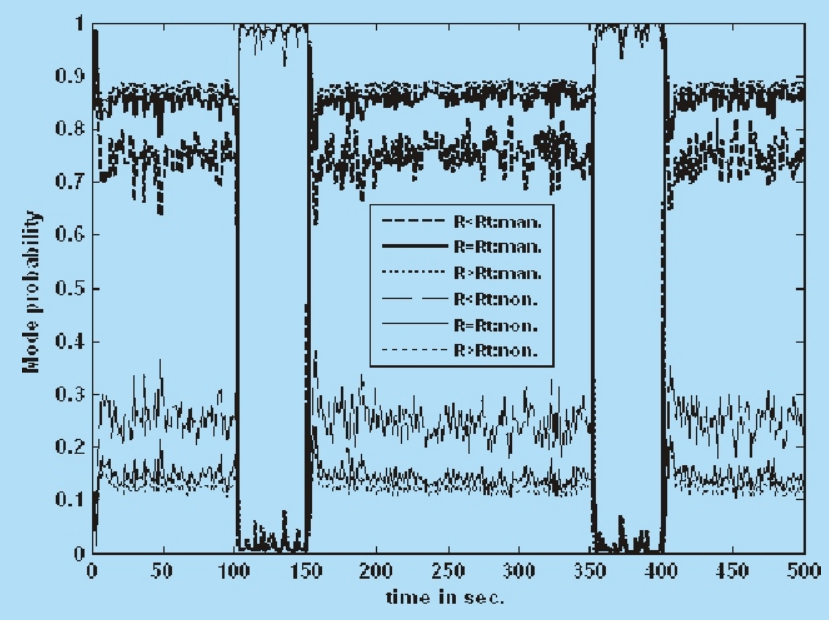

Figure 10 Mode probabilities - different $\mathrm{R}$

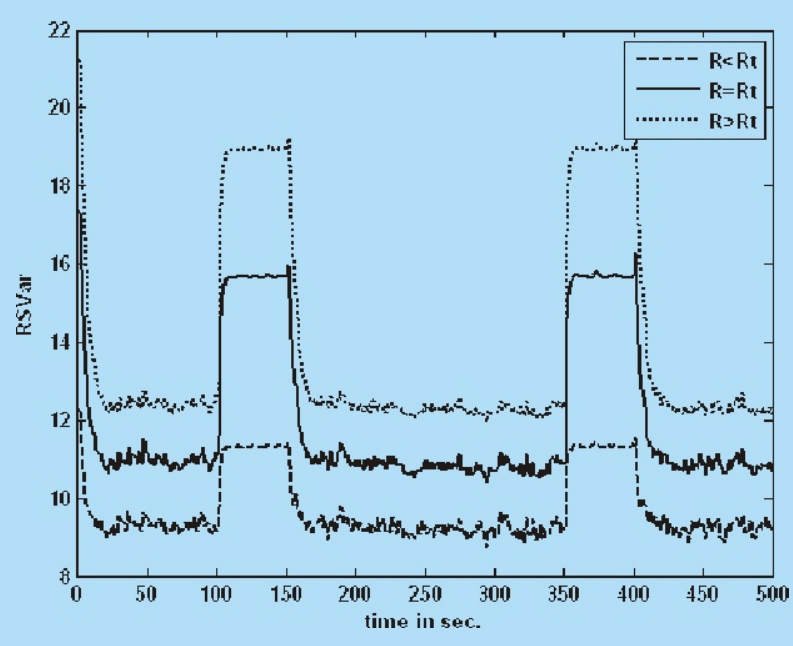

Figure 11 RSVar - different R 


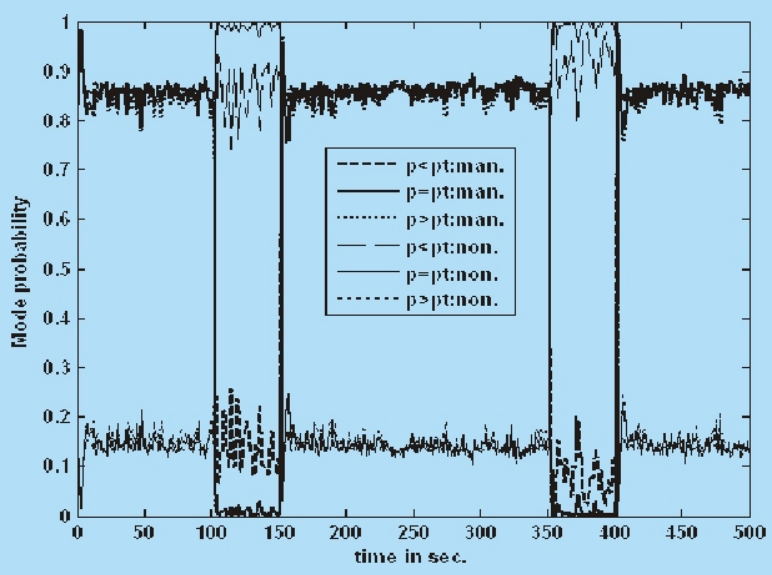

Figure 12 Mode probabilities - different

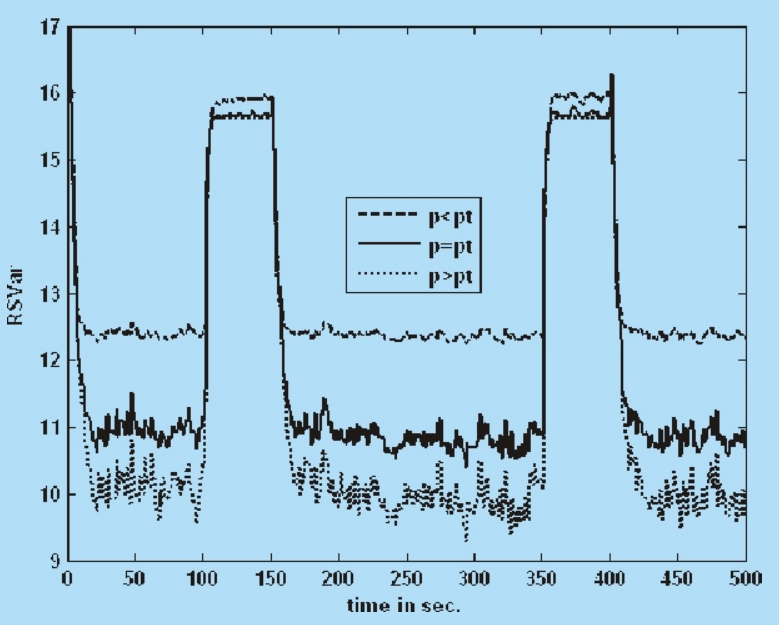

Figure 13 RSVar - different

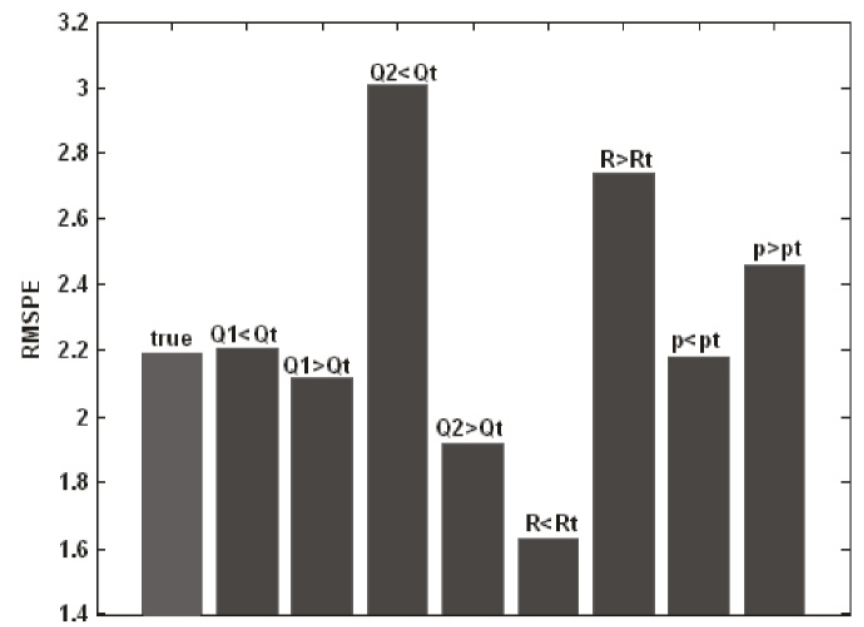

Figure 14a RMSPE - different parameters 


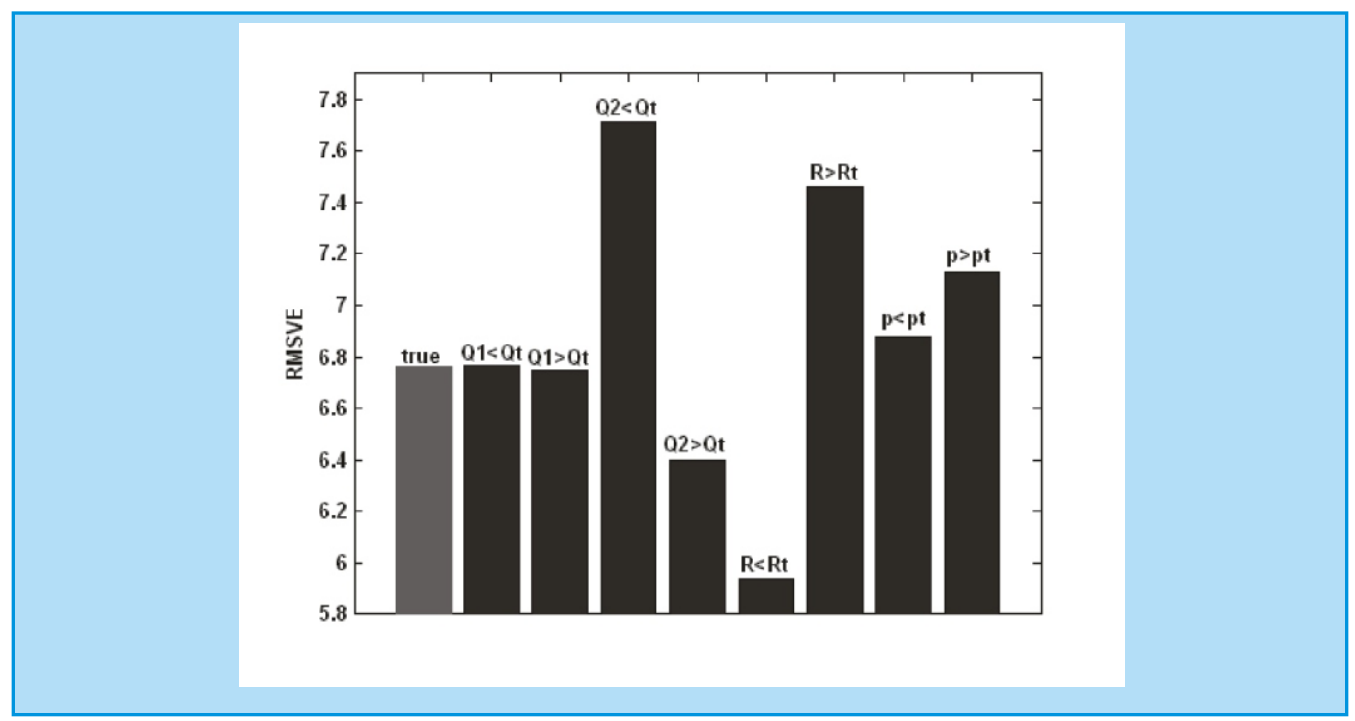

Figure 14b RMSVE - different parameters

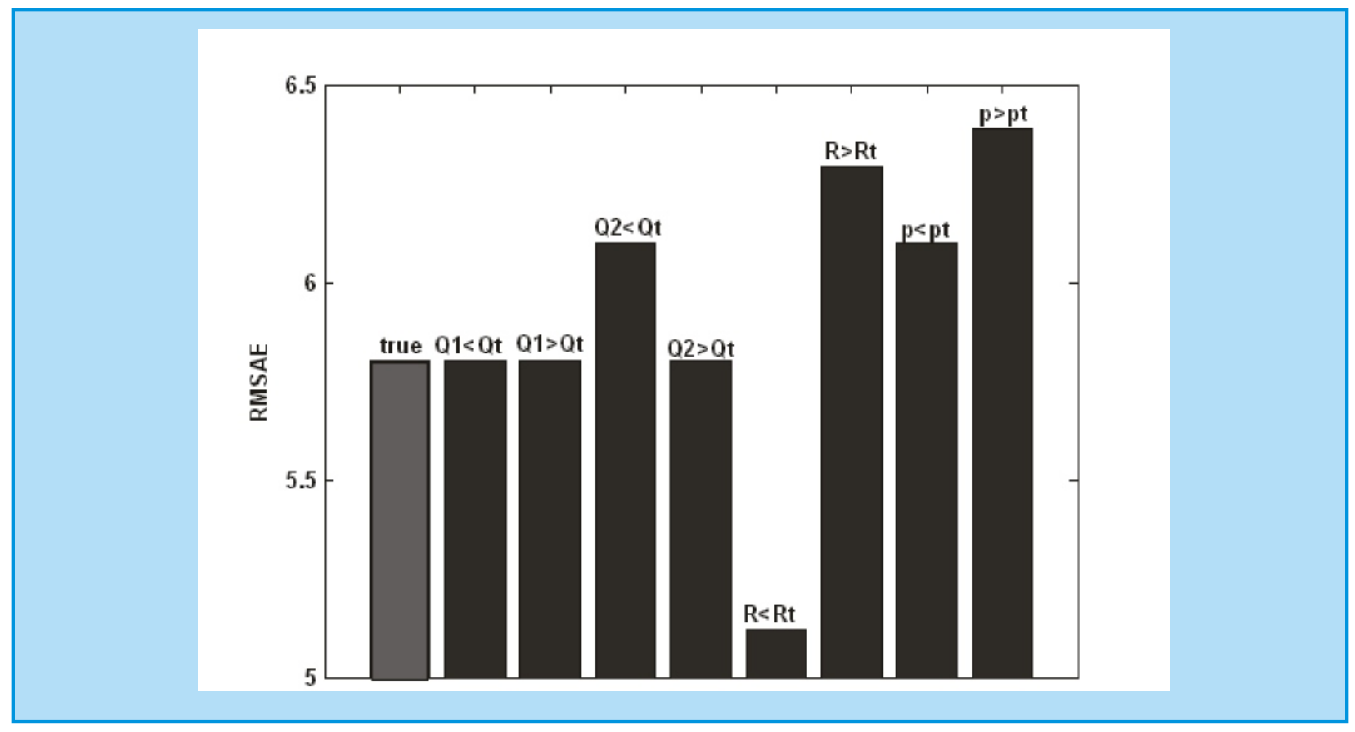

Figure 14c RMSAE - different parameters

\section{CONCLUDING REMARKS}

Sensitivity of conventional Kalman filter having constant velocity model, conventional Kalman filter having constant acceleration model and IMMKF for different process noise covariance, measurement noise covariance and model transition probabilities are investigated. Results showed that, IMMKF exhibits overall better performance and is more suitable for tracking maneuvering targets than conventional Kalman filters. The performance of IMMKF for different tuning parameters is explored. It is concluded that the chosen process noise variances higher than their true values gives the estimated states close to their true values at the same time the uncertainty in target state estimation is high. Similarly, measurement noise variance lesser than it's true, gives improved state estimation and reduced the associated uncertainty. Diagonal elements of model transition matrix derived from the sojourn time gives better results.

\section{REFERENCES}

1. Kalman RE, A New Approach to Linear filtering and prediction Problems, Trans. of the ASME, Journal of Basic Engineering, pp.35-45, March 1960.

2. Maybeck \& Peter S, Stochastic Models, Estimation and Control, Volume I, Academic Press, Inc., 1979.

3. Gelb A, Applied Optimal Estimation, MIT Press, Cambridge, Inc., 1974.

4. Grewal, Mohinder S, \& Angus P Andrews, Kalman Filtering Theory and Practice, Upper Saddle River, NJ USA, Prentice Hall, 1993. 
5. Jacobs OLR, Introduction to Control Theory, $2^{\text {nd }}$ Edition, Oxford University Press, 1993.

6. Brown RG, and PYC Hwang, Introduction to Random Signals and Applied Kalman Filtering, $2^{\text {nd }}$ Edition, Johan Wiley \& Sons, Inc., 1992.

7. IH Whang, J Lee \& T Sung, Modified Input Estimation Technique using Pseudoresiduals, IEEE Trans. Aero. and Elec. Sys., 30(1), pp.220-228, 1994.

8. YT Chan, AGC Hu \& JB Plant, A Kalman Filter Based tracking Scheme with Input Estimation, IEEE Trans. Aero. and Elec. Sys., 15(2), pp.237-244, 1979.

9. MS Woolfson, An Evaluation of Maneuver Detector Algorithms, GEC J. of Research, 3(3), pp.181-190, 1985.

10. F Gustafsson, Adaptive Filtering and Change Detection, Wiley, 2001.

11. H Lee \& MJ Tahk, Generalized Input-estimation Technique for Tracking Maneuvering Targets, IEEE Trans. Aero. and Elec. Sys., 35(4), pp.1388-1402, 1999.

12. PL Bogler, Tracking a Maneuvering Target using Input Estimation, IEEE Trans. Aero. and Elec. Sys., 23(3), pp.298-310, 1987.

13. Bar-Shalom. Y \& Xiao Rong Li, Multitarget Multisensor Tracking: Principles and Techniques, 1995 (ISBN 0-9648312-0-1).

14. Blom, HAP, \& Bar-Shalom Y, The Interacting Multiple Model Algorithm for Systems with Markovian Switching Coefficients, IEEE Trans. Aut. Con., Vol AC-33, pp780-783, Aug 1988.

15. E Mazor, A Averbuch, Y Bae-Shalom \& J Dayan, Interacting Multiple Model Methods in Target Tracking: A Survey, IEEE Trans. Aero. Elec. Sys., vol 34, pp103-23, 1998.
16. VPS Naidu, Girija G \& Shanthakumar N, Three Model IMM-EKF for Tracking Targets Executing Evasive Maneuvers, Paper no. AIAA2007-1204, AIAA Aerospace meeting and Exhibition, 8-11, Jan. 2007, Reno, Nevada.

17. X Rong Li \& Vesselin P Jilkov, "Survey of Maneuvering Target Tracking Part I: Dynamic Models", IEEE Trans. Aero. Elec. Sys., 39, pp.13331364, 2003.

18. E V Stansfield, Kalman Filters-Applications and Pitfalls, Kalman filter tutorial, Thales Research Ltd., Heckfield Place, $7^{\text {th }}$ March 2001.

19. Yaakov Bar-Shalom \& Xiao-Rong Li, Estimation and Tracking: Principles, Techniques and Software, Artech House, Bostan, 1993.

20. Iliyana Simeonova \& Tzvetan Semerdjiev, Specific Features of IMM Tracking Filter Design, Information \& Security, An International Journal, Vol 9, pp154$165,2002$.

21. VPS Naidu, N Shanthakumar \& Girija G, "Multisensor Multitarget (MSMT) State Estimation", NAL PD FC0310, May 2003.

22. Bloomer L \& Gray JE, Are More Models Better?: The Effect of the Model transition matrix on the IMM Filter, In the Southeastern Symposium on System theory (SSST), Hentsville, AL, March 2002.

23. VPS Naidu, Girija G \& J R Raol Evaluation of data association and fusion algorithms for tracking in the presence of measurement loss, paper no. AIAA 2003-5733, AIAA international Conference on Navigation, Guidance and Control, Austin, USA, August-2003. 